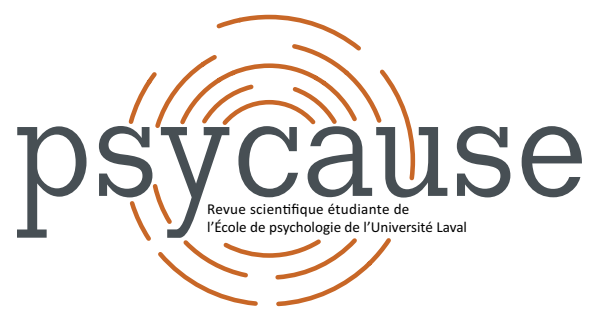

\title{
PSYCAUSE
}

Revue scientifique étudiante de l'École de psychologie de l'Université Laval

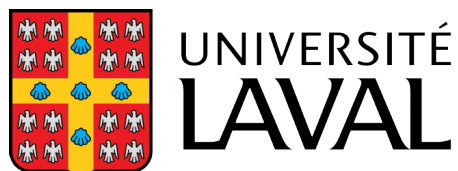

Faculté des sciences sociales École de psychologie

NOVEMBRE 2020 - VOL. $10 \mathrm{~N}^{\circ} 2$

\section{RÉSEAU SOCIAL ET CONFIGURATIONS IDENTITAIRES MULTICULTURELLES CHEZ LA DEUXIÈME GÉNÉRATION NORD-AMÉRICAINE}

Sarah COMTE ${ }^{1 * \forall}$, Florence DUHAIME ${ }^{1 \forall}$, Sarah GIRARD ${ }^{1}$, Jennifer TAING ${ }^{1}$ \& Maya YAMPOLSKY ${ }^{1}$

${ }^{1}$ École de psychologie, Université Laval

* sarah.comte.1@ulaval.ca

${ }^{\forall}$ Contribution équivalente des auteurices

\section{Pour citer l'article}

Comte, S., Duhaime, F., Girard, S., Taing, J., \& Yampolsky, M. (2020). Réseau social et configurations identitaires multiculturelles chez la deuxième génération nord-américaine Psycause: Revue scientifique étudiante de l'École de psychologie de I'Université Laval, 10(2), 25-26. 


\title{
RÉSEAU SOCIAL ET CONFIGURATIONS IDENTITAIRES MULTICULTURELLES CHEZ LA DEUXIÈME GÉNÉRATION NORD-AMÉRICAINE
}

\author{
Sarah COMTE ${ }^{1 *}$, Florence DUHAIME ${ }^{1}$, Sarah GIRARD ${ }^{1}$, Jennifer TAING ${ }^{1}$ \& Maya YAMPOLSKY ${ }^{1}$ \\ 1 École de psychologie, Université Laval \\ *sarah.comte.1@ulaval.ca \\ ${ }^{\forall}$ Contribution équivalente des auteurices
}

\section{Mots-clés : Identité multiculturelle, configurations identitaires, deuxième génération, réseau social}

L'identité culturelle est un construit multidimensionnel qui repose sur un sentiment de soi en tant que membre d'un groupe culturel (Berry, Phinney, Sam \& Vedder, 2006). Cette identité chez les individus de la deuxième génération ( $\left.2^{\mathrm{e}} \mathrm{G}\right)$ est particulière puisqu'ils doivent maintenir la culture d'origine transmise par leurs parents migrants, tout en grandissant dans la culture majoritaire du pays d'accueil. Le modèle cognitivo-développemental de l'intégration des identités sociales (CDSMII; Amiot, de la Sablonnière, Terry \& Smith, 2007) propose trois configurations identitaires, soit l'organisation cognitive des relations entre les identités à l'intérieur du concept de soi (Figure 1). La première est l'intégration, qui consiste à former une cohésion entre les identités étant perçues comme étant complémentaires. La seconde est la compartimentation, où les identités sont séparées, puisqu'elles sont perçues comme étant opposées. L'individu peut minimiser, réprimer ou éviter d'exprimer certaines identités culturelles selon les contextes socioculturels. La troisième est la catégorisation d'origine ou majoritaire, soit la présence prédominante et exclusive d'une seule identité culturelle.

Les identités multiculturelles se construisent de manière indissociable des relations sociales, d'où l'importance de s'intéresser au lien entre les deux. En effet, selon certains auteurs, la diversité ethnoculturelle au sein du réseau social et le degré d'interconnectivité entre les membres du réseau (proximité et liens) influence la manière dont un individu négocie ses identités culturelles (Repke \& Benet Martínez, 2017). Cependant, cette relation n'a jamais été validée empiriquement auprès de la $2^{\mathrm{e}} \mathrm{G}$ avec le CDSMII.

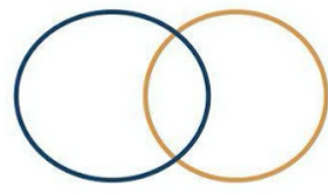

Intégration

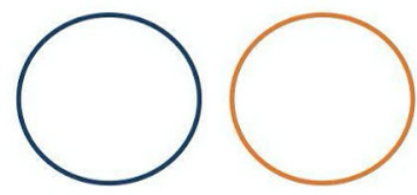

Compartimentation

L'objectif de cette étude est donc d'acquérir une compréhension plus nuancée du lien entre les structures des réseaux sociaux de l'individu (diversité culturelle des pairs et interconnectivité entre les pairs) et la configuration des identités culturelles d'origine et majoritaire dans le soi chez les adultes de la $2^{\mathrm{e}} \mathrm{G}$ en Amérique du Nord.

\section{Méthode}

Quatre-vingt-sept participants états-uniens, recrutés sur la plateforme ProlificAcademic, ont complété un sondage en ligne contenant des questions sociodémographiques et des items $^{1}$ mesurant les configurations identitaires (MULTIIS; Yampolsky, Amiot \& de la Sablonnière, 2016) ainsi que la structure des réseaux sociaux. La structure du réseau social a été analysée avec la procédure de Crossley et ses collègues (2015), c'est-à-dire en calculant l'interconnectivité (densité du réseau social et proximité relationnelle entre l'individu et son réseau) et la diversité culturelle (homogénéité ethnoculturelle des membres du réseau et similarité ethnoculturelle entre l'individu et son réseau).

\section{Résultats et discussion}

Des corrélations et régressions multiples ont été effectuées. La Figure 2 illustre la structure type du réseau social de quatre participants selon les configurations identitaires. Les résultats indiquent que plus les participants ont leurs identités intégrées (Figure 2d), plus ils sont proches de leurs alters (c.-à-d., les membres du réseau social) et plus ils ont tendance à partager une origine ethnoculturelle similaire avec eux. La proximité relationnelle est le seul prédicteur

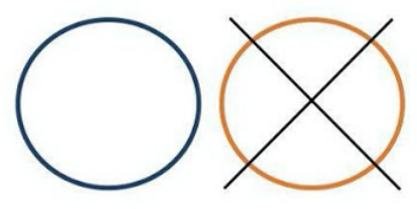

Catégorisation vers la culture d'origine

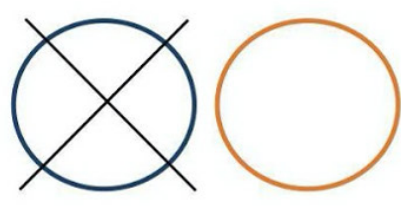

Catégorisation vers la culture majoritaire

Figure 1. Schéma des quatre configurations identitaires (la catégorisation vers la culture d'origine étant séparée de la catégorisation vers la culture majoritaire aux fins de l'étude). Chaque cercle représente une identité culturelle. Le cercle bleu désigne la culture d'origine et le cercle orange désigne la culture majoritaire (dans ce cas-ci, la culture états-unienne).

1 Exemple d'item issu du questionnaire: "Each of my cultural identities is a separate part of who I am. » 
(a) Participant catégorisé (origine)

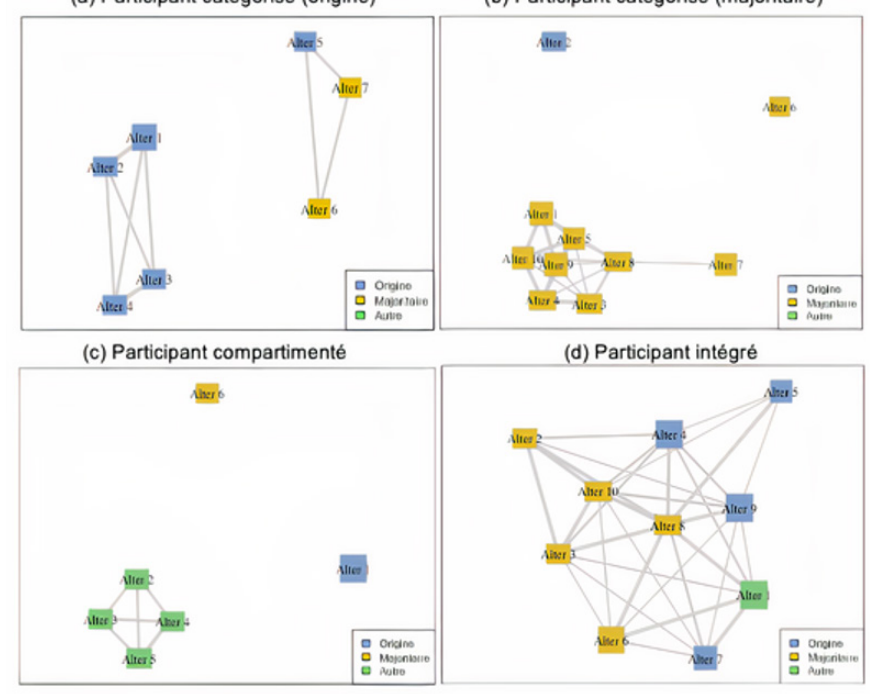

Figure 2. Schéma de la structure des réseaux sociaux de quatre participants correspondant aux quatre configurations identitaires. La proximité relationnelle des alters est représentée par la longueur et l'épaisseur des lignes (plus une ligne est courte et épaisse, plus les alters sont proches). Une absence de ligne signifie une absence de liens entre les alters. L'individu n'y est pas représenté.

significatif de l'intégration, ce qui suggère qu'entretenir de fortes relations avec ses alters serait essentiel pour l'intégration des identités culturelles (Repke \& Benet- Martínez 2017, 2019).

L'absence de résultat significatif pour la compartimentation (Figure 2c) pourrait être dû à la taille de l'échantillon $(N=87)$ qui ne permet pas d'avoir une quantité suffisante de participants compartimentés représentative dans l'échantillon. Or, les analyses descriptives révèlent que plus les participants sont compartimentés, moins ils ont d'alters dans leur réseau social, peu importe les origines ethnoculturelles. Ce manque de connexions sociales pourrait refléter l'expérience de marginalisation survenant lorsqu'un individu ne souhaite pas maintenir sa culture d'origine tout en évitant les interactions avec la société majoritaire plus large (Berry \& Hou, 2017).

En ce qui concerne la catégorisation d'origine (Figure 2a) ou majoritaire (Figure 2b), aucun résultat significatif n'a été observé avec l'interconnectivité du réseau. Toutefois, les participants catégorisés vers la culture d'origine ont plus d'alters de leur culture d'origine, contrairement aux participants catégorisés vers la culture majoritaire. Les participants catégorisés vers la culture d'origine pourraient avoir un plus grand niveau d'engagement envers leur culture d'origine que les participants catégorisés vers la culture majoritaire, menant à un plus fort sentiment d'appartenance et d'identification à cette culture (Ferguson, Costigan, Clarke \& Ge, 2016).
L'approche des réseaux sociaux permet d'enrichir les théories et modèles psychologiques comme le CDMSII (Amiot et coll., 2007; Yampolsky et coll., 2016) en incluant les relations sociales de la 2e G (Repke \& Benet-Martínez, 2019), menant à une meilleure représentation de leur réalité sociale et culturelle. Cette étude révèle des enjeux pertinents des personnes de $2^{\text {e }} \mathrm{G}$ vivant dans une société majoritaire en soulignant l'importance d'explorer d'autres aspects de la relation entre les réseaux sociaux et les configurations identitaires tels que la qualité des relations entretenues et le sentiment d'appartenance.

\section{Références}

Amiot, C. E., de la Sablonnière, R., Terry, D. J., \& Smith, J. R. (2007). Integration of Social Identities in the Self: Toward a CognitiveDevelopmental Model. Personality and Social Psychology Review, 11(4), 364-388. http://doi.org/10.1177/1088868307304091

Berry, J. W., Phinney, J. S., Sam, D. L., \& Vedder, P. (2006). Immigrant Youth: Acculturation, Identity, and Adaptation. Applied Psychology, 55(3), 303-332. http://doi. org/10.1111/j.1464-0597.2006.00256.x

Berry, J. W., \& Hou, F. (2017). Acculturation, Discrimination and Wellbeing Among Second Generation of Immigrants in Canada. International Journal of Intercultural Relations, 61, 29-39. http://doi.org/10.1016/j.ijintrel.2017.08.003

Crossley, N., Bellotti, E., Edwards, G., Everett, M. G., Koskinen, J., \& Tranmer, M. (2015). Social Network Analysis for EgoNets: Social Network Analysis for Actor-Centred Networks. Londres, Royaume-Uni: SAGE Publications.

Ferguson, G. M., Costigan, C. L., Clarke, C. V., \& Ge, J. S. (2016). Introducing Remote Enculturation: Learning Your Heritage Culture from Afar. Child Development Perspectives, 10(3), 166-171. http://doi.org/10.1111/cdep.12181

Repke, L., \& Benet-Martínez, V. (2017). Conceptualizing the Dynamics Between Bicultural Identification and Personal Social Networks. Frontiers in Psychology, 8(469), 1-19. http://doi.org/10.3389/fpsyg.2017.00469

Repke, L., \& Benet-Martínez, V. (2019). The Interplay Between the One and the Others: Multiple Cultural Identifications and Social Networks. Journal of Social Issues, 75(2), 436459. http://doi.org/10.1111/josi.12323

Yampolsky, M. A., Amiot, C. E., \& de la Sablonnière, R. (2016). The Multicultural Identity Integration Scale (Multiis) : Developing a Comprehensive Measure for Configuring One's Multiple Cultural Identities Within the Self. Cultural Diversity and Ethnic Minority Psychology, 22(2), 166-184. http://doi. org/10.1037/cdp0000043

\section{Pour citer l'article}

Comte, S., Duhaime, F., Girard, S., Taing, J., \& Yampolsky, M. (2020). Réseau social et configurations identitaires multiculturelles chez la deuxième génération nord-américaine Psycause: Revue scientifique étudiante de l'École de psychologie de I'Université Laval, 10(2), 25-26. 